\title{
PERFIL DE LAS PERSONAS SIN HOGAR QUE HABITAN EN CASAS DE ACOGIDA
}

\author{
Ana María Velasco Pinchete \\ Educadora Social \\ anamavepi@gmail.com \\ Ana Isabel Isidro de Pedro
}

Profesora Titular de E.U. Departamento de Psicología Social y Antropología Universidad de Salamanca anyis@usal.es

Fecha de Recepción: 6 Febrero 2019

Fecha de Admisión: 30 Abril 2019

\section{RESUMEN}

El sinhogarismo no es un fenómeno ajeno a nuestra sociedad y la mayoría de las personas sin hogar que llevan un tiempo en esta situación se convierten en personas altamente vulnerables y en manifiesta exclusión social, hecho que dificulta las intervenciones profesionales conducentes a su reinserción. Una de las medidas transitorias son las casas de acogida, un tipo de recurso de residencia de larga duración, aunque temporal. En el presente trabajo se analiza, desde una perspectiva socio-educativa, el perfil de las personas sin hogar que habitan en casas de acogida españolas. Para ello, se realiza una investigación cualitativa -siguiendo la metodología de historias de vidasobre una muestra de personas sin hogar residentes en casas de acogida, concretamente en el municipio de Fuenlabrada (Madrid), con edades comprendidas entre los 23 y 67 años. En este sentido, encontramos que los resultados, en esencia, concuerdan con los estudios previos hallados en la literatura. Por ejemplo, las causas por las que se encuentran en las casas de acogida suelen ser: situación económica precaria, carencia de empleo y de recursos económicos alternativos, una escasa o nula red social y la dificultad para hablar español. Reconocemos que el proceso de reinserción en la sociedad de estas personas es largo y laborioso en cuanto a esfuerzos por parte de los equipos multidisciplinares. Por ello, se proponen unas pautas de intervención psicosocial y educativa, que pueden contribuir a la reinserción de este colectivo.

Palabras clave: personas sin hogar; casas de acogida; perfil; exclusión social

\section{ABSTRACT}

Homeless people s profile who live in shelters. Homelessness is not a phenomenon indifferent to our society and most of the homeless people who have been in this situation for a long time become highly vulnerable people and in manifestly socially excluded situation, a fact that make dif- 
ficult professional interventions leading to their reintegration. One of the transitory measures is shelters, a type of long-term residence resource, but temporary. In the present work, the profile of homeless people living in Spanish shelters is analyzed from a socio-educational perspective. To do this, a qualitative research is conducted -following the life stories methodology- on a sample of homeless people living in shelters, specifically in the town of Fuenlabrada (Madrid, Spain), aged between 23 and 67 years. In this sense, we find that the results, in essence, agree with the previous studies found in the literature. Thus, the reasons why they are found in shelters are usually: a precarious economic situation, a lack of both employment and alternative economic resources, a little or absent social network and their difficulty to speak Spanish. We recognize that the process of reintegration of these people into the society is long and laborious in terms of efforts from multidisciplinary teams. For this reason, some psychosocial and educational intervention guidelines are proposed, which can contribute to the reintegration of this collective.

Keywords: homeless people; shelters; profile; social exclusion

\section{JUSTIFICACIÓN TEÓRICA}

Para poder llevar a cabo y entender el siguiente trabajo debemos diferenciar los tipos de pobreza, siguiendo a Malgesini y Canadijala (2014): pobreza absoluta o severa (cuando las necesidades básicas como la alimentación no están cubiertas) y pobreza relativa (se obtienen los ingresos económicos básicos para satisfacer alguna de las necesidades primordiales básicas). Y al término de pobreza es importante añadir el término de desigualdad porque si no hubiera desigualdad entre las clases sociales se concluye que no habría pobreza. Según Naguera (2004), la desigualdad tiene que ser entendida como las diferencias entre los beneficios y los gastos vinculados a la satisfacción de necesidades básicas, además de la diferencia entre las clases sociales.

Así, por todas las desigualdades existentes, podemos distinguir entre distintos ámbitos de exclusión social como el económico, laboral, formativo, socio-sanitario, relacional, de la ciudadanía y participación y residencial. Dadas las características del trabajo, nos centraremos en la exclusión social de carácter residencial. En este sentido, según el Informe FEANTSA (Federación de Asociaciones a Favor de las Personas Sin Techo, 2008), la vivienda y las políticas sociales asociadas a ella son la principal causa de sinhogarismo. Este informe reconoce que existen cinco factores que pueden producir la situación de sinhogarismo, a saber: 1) la vivienda no es asequible (tanto compra como el alquiler); 2) la falta de disponibilidad de casas tanto en el sector público como en el privado; 3) que la vivienda no tenga unos mínimos estándares de calidad, de manera que vivir en la misma se puede considerar exclusión residencial; 4) el hacinamiento, es decir, que haya muchas personas viviendo en esa casa y no se den las condiciones necesarias para que sea una vivienda digna; 5) desahucios, que en los últimos años han crecido fuertemente en España.

FEANTSA, a través de European Tipology on Homelessness (Índice ETHOS) (2008), clasifica la tipología de personas sin hogar y la exclusión social residencial a través de cuatro ejes importantes: personas sin techo, personas sin vivienda (con un lugar para dormir pero temporal en instituciones 0 albergues), personas residiendo en viviendas inseguras y personas residiendo en viviendas inadecuadas. Partiendo de esta tipología, nos centraremos en el rango de personas sin vivienda y, dentro de ella, en personas que habitan en alojamientos de emergencia.

Según la nota de prensa del INE (2012), en España había 22938 personas sin hogar en el año 2012, mayores de edad, que por diferentes condiciones, habían llegado a vivir en la calle.

Para solucionar el problema de las personas sin hogar existen asociaciones, ONGs y otras instituciones que se encargan de administrar diferentes recursos para paliarlo. Entre dichos recursos destacan dos grandes grupos: los albergues y las casas de acogida. 
Según Bravo y Rebollo (2005), las casas de acogida son un recurso especializado y su objetivo es acoger temporalmente a personas con diversas problemáticas con el objetivo de establecer unos objetivos y unas metas para que su situación problemática desaparezca y, así, poder reinsertarlas en la sociedad.

Claramente, para llevar a cabo la reinserción de las personas, debemos implementar una metodología eficaz y, aunque no existe mucha información sobre las casas de acogida y cómo se debe intervenir en ellas, contamos con el método de intervención no definido que se usa en la Asociación San Ricardo Pampuri (en la cual se desarrolla la investigación de este trabajo), método que se lleva a cabo a través de las siguientes actividades:

Entrevista motivacional: Entrevista para destacar las actitudes y aptitudes de la persona y mostrar sus fortalezas y la ayuda de los compañeros.

Valoración de las necesidades: Una vez realizada la motivación, se lleva a cabo la detección de necesidades 0 carencias que el usuario presenta para poder cubrirlas y ayudarle a erradicarlas.

Creación de un compromiso de trabajo: Se establece un compromiso con el usuario para poder crear una alianza y en este momento se conoce el entorno, los problemas, los recursos y los posibles riesgos que pudieran existir.

Planteamiento de metas: Una vez conocido el contexto, se establecen con el sujeto una serie de metas para conseguir su reinserción en la sociedad de manera efectiva.

Acompañamiento psicológico, socio-educativo y jurídico: En ningún momento los usuarios pueden sentirse solos, siempre existe un educador, psicólogo o trabajador social que trabaja con ellos para conseguir la reinserción. También se trabaja junto a abogados, jueces y policías (Policía Nacional, Policía Local y Guardia Civil) para solventar problemas, si existiesen.

Entrenamiento en las habilidades sociales, habilidades laborales, habilidades paterno-filiales, etc.: Realización de diferentes actividades para mejorar las habilidades sociales, automotivación, autoestima... Siempre pensadas desde la detección de necesidades.

Abandono de drogodependencias: En algunos casos se encuentran problemas subyacentes y, para intervenir, se cuenta con el apoyo de otras instituciones especializadas en estas cuestiones.

Formación: Uno de los objetivos más importantes es formar a las personas, porque el $98 \%$ de los usuarios no tienen más que formación básica y se pretende que, a través de cursos, puedan formarse y llegar a trabajar en el empleo que decidan.

Solicitar los recursos con los que puedan contar: Se les ayuda con los trámites burocráticos que deben llevar a cabo.

Retomar su preocupación por su salud: En el momento en el que las personas entran en la casa se encuentran en una situación vulnerable y ello hace que se despreocupen por aspectos de la salud que no tienen una gran importancia. En algunos de los casos se ha debido reeducar a la persona en la higiene corporal.

\section{OBJETIVOS}

Los objetivos aquí pretendidos, son fundamentalmente:

Conocer las características del colectivo y el motivo por el que se encuentran en esa situación.

Establecer unas pautas de intervención con el colectivo de personas sin hogar.

\section{METODOLOGíA}

La investigación realizada sigue una metodología cualitativa que según, Rodríguez, Gil y García (1996), estudia la realidad en su contexto natural, es decir, se estudia en el momento que pasa y es capaz de interpretar los fenómenos de manera adecuada para darle sentido, teniendo en cuenta a las 
personas implicadas. Esta técnica incluye la recogida de datos a través de distintos soportes como pueden ser historias de vida, imágenes... que describen una situación en concreto y dan significado a la vida de las personas. Y, concretamente, dentro de esta metodología cualitativa usaremos la metodología de "historias de vida". Según Ruiz Olabuénaga (2012), las historias de vida son la mejor forma que permite al investigador acceder a observar cómo los investigados crean y reflejan el mundo social en el que habitan. Además, ofrece un marco interpretativo a través de la experiencia que han sentido y sufrido los usuarios de la misma.

Una vez recogidos los datos, éstos han sido analizados mediante el programa de Software NVIV0-11 que, Según Gutiérrez, Palacios y Sánchez (2013), está programado para analizar datos cualitativos que proceden de las historias de vida, etc. y permite trabajar con una gran variedad de formatos (ficheros de audio, videos... además de textos).

\section{RESULTADOS}

Se ha considerado adecuado presentar los datos divididos por género, es decir, por un lado, hombres y, por otro, mujeres, debido a que los hombres no presentan cargas familiares y las mujeres sí.

\section{CASA DE HOMBRES}

En el análisis del género masculino, contamos con cinco integrantes en la casa, todos ellos mayores de edad. La procedencia mayoritaria es de origen marroquí, con dos personas, un español, un cubano con su nieto y un subsahariano. Las edades entre las que se encuentran oscilan entre los 23 y 67 años, predominando los 56 ya que dos de los usuarios tienen esa edad y el último tiene 58.

En la siguiente nube de palabras, encontramos las que más se repiten entre los cinco integrantes de la casa.

Figura 1. Resultados obtenidos del género masculino presentados en forma de nube de palabras

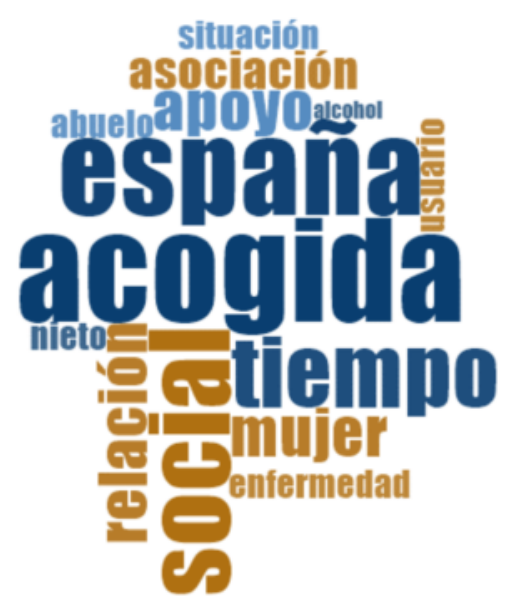

Como podemos observar en la Figura 1, las palabras que salen en un tamaño mayor y en azul oscuro son las palabras más repetidas en las historias de vida de los usuarios. La primera palabra que nos aparece es "acogida" porque, gracias al recurso en el que se encuentran, pueden acceder a una calidad de vida estable y si no estuvieran en la casa, vivirían en la calle. A su vez, esta palabra 
está relacionada con "asociación", la cual aparece más pequeña pero bastante frecuente entre los usuarios. Destaca la palabra "España”, porque la mayoría de los integrantes de la casa (el 80\%) son de origen extranjero y la llegada a España supuso un cambio en sus vidas, ya que ellos buscaban mejorar su calidad de vida.

A continuación, las palabras aparecen en un color marrón oscuro y aclarándose a medida que va bajando la frecuencia de uso en el relato. Dos palabras que se deben analizar unidas son "social" y "relación", las cuales se refieren a las relaciones, es decir, al grupo de apoyo -como es la familia-, el grupo de iguales y los compañeros con los que cuentan. Estas palabras destacan porque, por desgracia, carecen de un apoyo social estable al encontrarse en un país diferente y lejos de su familia y amigos. En el caso del usuario de origen español, su red social es también escasa por diferentes motivos como su separación matrimonial y el alcoholismo que padece.

Otra de las palaras que destaca es "mujer", porque el $60 \%$ de los usuarios mantienen una relación estable con una mujer, aunque dicha relación sea a distancia y no puedan verse porque ellas no se encuentran en España. Las relaciones con ellas son satisfactorias y placenteras. Mantienen un contacto telefónico habitual, es decir, hablan más de dos veces por semana con ellas por teléfono y también establecen relación constante a través de la aplicación WhatsApp. El 20\% de los usuarios mantiene una relación tensa con su exmujer, aunque en los momentos difíciles obtiene apoyo de ella y de su antigua unidad familiar (su exmujer y su hija). El 20\% restante, no mantiene relaciones estables con mujeres sino esporádicas y, por ello, no puede encontrar apoyo en ellas.

También destaca la palabra "enfermedad" porque el $80 \%$ de los usuarios tienen una patología. Un usuario padece cáncer y lleva a cabo tratamiento de quimioterapia y radioterapia, lo que le impide poder encontrar un trabajo y salir de la situación en la que se encuentra. El $40 \%$ tienen enfermedades que no les limitan a la hora de llevar a cabo actividades de la vida cotidiana, como es diabetes y problemas reproductivos. Finalmente, otro usuario sufre una enfermedad difícil, el alcoholismo, en la que es muy complicado y muchas veces imposible compaginar con una vida personal, social o laboral. Le cuesta mantenerse sobrio, aunque recibe ayuda por parte de diferentes asociaciones y profesionales.

Con la palabra "apoyo" hacen referencia a la ayuda que se les proporciona desde la Asociación ya que, como se ha analizado anteriormente, disponen de una red de apoyo escasa o nula en España. Por ello, la Asociación tiene a dos voluntarios que pasan mucho tiempo haciéndoles compañía y ayudándolos cuando lo necesitan, para que no se sientan solos. Además, la Asociación pone a su disposición un equipo de profesionales multidisciplinares como son psicólogos, educadores sociales, integradores sociales y trabajadores sociales.

La última palabra que es importante en este bloque es "abuelo", que se refiere a uno de los usuarios que habita en la casa. Es el responsable de la misma cuando no hay supervisión directa por parte del equipo y se encarga de la casa y de la gestión de los desencuentros que pueda haber en ella, comunicándoselos directamente a los profesionales. Es una de las personas más importantes de la casa debido a su alta responsabilidad y trabajo en equipo cuando los profesionales se lo requieren.

Como se puede observar, el colectivo carece de apoyos fuertes fuera de la casa debido, fundamentalmente, a la lejanía de sus lugares de origen y al padecimiento de drogodependencias y/o enfermedades que han entorpecido o impedido las relaciones afectivas de los usuarios

\section{CASA DE MUJERES}

A continuación, se presenta el análisis de la casa de mujeres que está integrada por siete personas del género femenino, con sus respectivos hijos o hijas que las acompañan en todo momento. La nube de palabras representa las palabras más utilizadas por las usuarias. 
Figura 2. Resultados obtenidos del género femenino presentados en forma de nube de palabras.

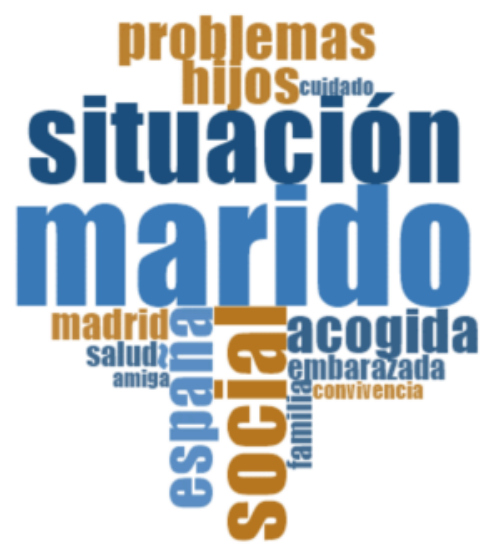

La palabra que más se repite es "marido". Esta palabra divide a las usuarias en dos grupos diferenciados:

El marido la apoya: solo el $28,58 \%$ de las usuarias recibe apoyo de sus parejas y padres de sus hijos. Es decir, aunque su pareja no conviva con ellas ni esté en España siguen manteniendo un fuerte vínculo entre ellos. Mantienen contacto más de dos veces por semana y ellas tienen la ilusión de que pronto podrán llevar a cabo una reunificación familiar en España.

El marido no la apoya: el $71,42 \%$ de las usuarias no recibe apoyos de sus maridos, exmaridos o parejas actuales. Ellas son personas fuertes, que tienen asumir del rol de madre y padre a la vez. Además, un $14,28 \%$ de las usuarias ha recibido maltrato físico o psicológico por parte de su pareja 0 del núcleo familiar del marido. Otro $14,28 \%$ se ha convertido en una persona 'nula'; es decir, su marido cuando se la encuentra por la calle no la saluda ni le dirige la palabra, aunque esporádicamente realiza llamadas telefónicas para saber en qué situación se encuentran ella y sus hijos.

Otra de las palabras más usadas por las usuarias es "situación", porque se refieren al momento en el que se encuentran y no saben cómo salir de este bache.

La siguiente palabra es "hijos" refiriéndose a los niños que tienen a su cargo y la importancia que, para ellas, tienen sus hijos y los vínculos familiares que existen. El $85,72 \%$ de las usuarias tiene establecida con sus hijos una relación fuerte y recíproca; es decir, los hijos reconocen la importancia de sus madres y muestran afecto y cariño por ellas. Mientras que una usuaria expone que la maternidad es algo difícil para ella y prefiere que su hija viva en una casa de acogida para niños antes que con ella, ya que no es capaz de llevar a cabo las funciones de madre y compaginarlas con una vida social placentera.

Relacionado con los hijos, se debe hablar de las palabras "cuidados" y "salud" porque el $42,84 \%$ de las usuarias tienen hijos con diferentes problemas de salud (como parálisis cerebral) y esto condiciona la vida de las madres, quienes tienen que llevar a cabo un especial cuidado con ellos. El $28,56 \%$ de las usuarias con hijos con problemas son capaces de realizar todas las pautas médicas prescritas a sus hijos y las llevan a cabo de una manera adecuada y precisa para influir de manera positiva y beneficiosa en la salud de los hijos enfermos. Mientras que una usuaria decidió entregar a su hija a una casa de acogida para niños porque ella no se veía capacitada para seguir sosteniendo la guardia y custodia de la niña. 
En la palabra "social" se advierte una gran similitud con los usuarios de la casa de hombres porque se encuentran sin un apoyo ni una red social estable con la que poder contar en España, solo tienen a sus compañeras de casa y las relaciones que establezcan en las actividades que llevan a cabo. También hay que relacionarlo con la palabra "amiga", porque aunque en la actualidad el $85,72 \%$ no mantengan este tipo de relación, las amigas fueron las que las acogieron cuando no tenían donde vivir, aunque eso significara vivir en un salón, tener que conseguir comida o tener que realizar diferentes tareas para poder seguir viviendo en la casa.

La palabra "España" está relacionada con la llegada a nuestro país, buscando una mejor calidad de vida. El 42,84\% de las usuarias vinieron para mejorar la calidad de vida de sus hijos porque en su país de origen no existían los medios necesarios para cubrir las necesidades básicas de los niños enfermos. El 42,84\% de las usuarias Ilegaron a España para mejorar su propia calidad de vida. Un $28,56 \%$ vinieron luchando duramente frente a las adversidades que se encontraban en el camino y, una vez establecidas en España, formaron su familia. Un 14,28\% Ilegaron engañadas por familiares 0 amigos que les prometieron que venían a estudiar y al llegar se convirtieron en esclavas de la limpieza de las casas, trabajando explotadas en los negocios que la familia tenía. Una usuaria vino engañada por su marido, prometiéndole una vida mejor para formar su familia y ser feliz en España.

Destaca la palabra "Madrid" porque el $28,56 \%$ de las mujeres no tenían la idea inicial de acabar en Madrid (antes de empezar el viaje el 14,28\% tenía previsto vivir en Barcelona y el otro 14,28\% en Sevilla). Mientras que el $71,44 \%$ sí traían la idea de sus países de origen de residir en Madrid, por tener diferentes recursos a los que acceder.

Para ellas es importante la palabra "acogida" porque, como comentaban en los talleres y en las entrevistas individuales, la acogida es esa segunda oportunidad que han tenido en España para poder conseguir una cierta calidad de vida y desarrollarse personal y socialmente como miembros de la sociedad.

Destaca la palabra "embarazada" porque todas las usuarias tienen hijos y el $57,16 \%$ de la muestra tuvieron a sus hijos en España, mientras que el 42,84\% tuvieron a sus hijos en su país de origen y debieron trasladarse con ellos a España para poder obtener una mejora de la calidad de vida de niños y tratamiento médico que les ayudara a estar estables y produjera una mejoría en su salud.

Por último, analizaremos la palabra "convivencia". Al respecto, se ha de señalar la dificultad que existe en la convivencia con personas ajenas y que conoces una vez que entras en un recurso como es la casa de acogida la cual, además, tiene unas normas de obligado cumplimiento. Y, sin embargo, es de destacar la implicación de las usuarias al integrarse y cumplir las normas que se exigen de la mejor manera posible y facilitar la convivencia entre ellas, evitando, de manera eficiente, el surgimiento de problemas mayores y que las dificultades solo sean las propias de una convivencia cercana, como pueden darse en cualquier hogar normalizado con varios miembros conviviendo.

La diferencia que se puede extraer de las mujeres respecto a los hombres son los problemas de convivencia que se han ocasionado entre las usuarias. Debe entenderse que, en las casas de acogida dedicadas a mujeres, ellas viven con sus hijos y cada una tiene con sus hijos una relación afectiva y educativa muy diferente a la de su compañera y eso acarrea problemas, aunque no serios, en la convivencia cotidiana, pero en los que ha de intervenir el equipo multidisciplinar. También, se puede observar que la impronta y la influencia de sus respectivas culturas son muy fuertes y ello se trasluce en sus hábitos, por ejemplo, comer sin utilizar cubiertos, hablar con un tono de voz excesivamente alto, etc.

Otro de los elementos importantes que encontramos es la dependencia que muestran del marido o cónyuge. Este aspecto se trata en talleres específicos, aunque se cuenta con la importante limitación u obstáculo de las culturas propias y su fuerte influencia en la forma de ver la sociedad y manejarse en ella. 


\section{CONCLUSIONES}

A través de la investigación realizada, se puede establecer un perfil de las personas que se ven abocadas a formar parte del grupo de personas sin hogar y sus características comunes, las cuales se pueden encontrar en la siguiente descripción. Las personas sin hogar que nos encontramos en el municipio de Fuenlabrada, en la Asociación San Ricardo Pampuri, son personas jóvenes, de edades comprendidas entre 23 y 67 años, de origen fundamentalmente extranjero, sobre todo de países pertenecientes al continente africano. El tiempo medio que permanecen en la casa suele ser de 3,5 años, aunque se pueden observar casos que llevan más de 10 años. Se trata de personas que en su mayoría (el 66,67\%) padecen una enfermedad o tienen a su cargo descendientes directos con problemas graves de salud.

Las causas por las que se encuentran en esta situación se deben a dos grandes factores. El primero se refiere a la situación económica, ya que por diversos motivos como la escasa oferta de empleo (a veces combinado con problemas de salud), los usuarios no tienen trabajo ni disponen de medios económicos alternativos para poder sustentar los hogares en los que vivían. A esto, se le suma el segundo motivo, que es la escasa o nula red de apoyo social con el que cuentan en España, lo cual hace que empeore la situación en la que se encuentran y deban ejercer la mendicidad para poder sobrevivir, antes de saber que existen casas de acogida y que pueden acceder a ellas. Además, presentan escasas habilidades sociales e interpersonales que les condicionan a la hora de relacionarse con los demás. A lo que se le une la dificultad del idioma, porque la mayoría de las personas, no saben hablar español hasta que no llevan un cierto tiempo en España

Se puede asegurar que el proceso de reinserción en la sociedad de estas personas es largo y laborioso en cuanto a esfuerzos por parte de los equipos multidisciplinares. No obstante, para conseguir el proceso de reinserción social plena desde la Educación Social se puede llevar a cabo una importante labor con el colectivo a través de:

Alfabetización en la lengua oficial del país, es decir, enseñanza del castellano para poder facilitar la comunicación con los demás y la integración.

Talleres de habilidades sociales, a través de los cuales se fomente la escucha activa, la asertividad, etc.

Realización con los usuarios de un proyecto de vida, que consiste en la planificación en base a un objetivo, teniendo en cuenta las experiencias vividas para llevar a cabo un desarrollo óptimo de la persona.

Ofrecer ayuda y acompañamiento para realizar diferentes trámites necesarios.

Redactar un Curriculum Vitae y una carta de presentación y promover, a través de la orientación laboral, una búsqueda activa de empleo para facilitarles el acceso a la vida laboral.

Realizar actividades orientadas a la disciplina positiva, asertiva y democrática con los hijos.

Diagnosticar problemas relativos a los ámbitos familiar, laboral y social con el objetivo de gestionarlos adecuadamente y solventarlos.

Realizar un acompañamiento socio-educativo y psicosocial a las mujeres que han sufrido Mutilación Genital Femenina y ayudarlas a afrontarlo.

Abordar de manera funcional temas significativos como la violencia de género, bullying, sexting, etc.

Intervención inmediata en problemas como la violencia de género, a través de la ayuda de equipos multidisciplinares y organismos oficiales.

Promover campañas de sensibilización a toda la población para que se conozca el colectivo y disminuya sus prejuicios. 


\section{REFERENCIAS BIBLIOGRÁFICAS}

Bravo, C. y Rebollo, I. (2005). Casas de acogida: Desde la experiencia a la reflexión. Cuadernos de Trabajo Social, 18, 317-332.

FEANTSA (2008). El papel de la vivienda en el sinhogarismo. Recuperado de: http://www.feantsa.org/download/08_european_report_feantsa_housing_final_es70741158485 78375806.pdf

INE (2012). Encuesta sobre las personas sin hogar. Recuperado de: http://www.ine.es/dyngs/INEbase/es/operacion.htm?c=Estadistica_C\&cid=1254736176817\&m enu=ultiDatos\&idp=1254735976608

Malgesini, G. y Candalija, J. (2014). Dossier pobreza de EAPN España. Recuperado de: http://ibdigital.uib.es/greenstone/collect/portal_social/archives/eapn0017.dir/eapn0017.pdf

Noguera, J. A. (2004). Sobre el concepto de desigualdad en ciencias sociales. Revista de Ciencias Sociales 9, 1-18.

Palacios B., Gutiérrez A., y Sánchez M. C. (2013). NVIVO una herramienta de utilidad en el mundo de la comunicación. En M. Vicente, T. González y M. Pacheco (Coords.), Investigar la comunicación hoy. Revisión de políticas científicas y aportaciones metodológicas: Simposio Internacional sobre Política Científica en Comunicación (pp. 1003-1018). Valladolid: Universidad de Valladolid.

Rodríguez G., Gil J. y García E. (1996). Metodología de la investigación cualitativa. Granada: Aljibe.

Ruiz Olabuénaga J. (2012). Metodología de la investigación cualitativa. Bilbao: Universidad de Deusto. 
